\title{
Translational Virology in the Age of Pandemics
}

\author{
Kenneth Lundstrom* \\ Pan Therapeutics, Lutry, Switzerland
}

Keywords: pandemics, translational virology, basic research, preclinical studies, clinical trials, drug and vaccine approval

\section{THE WAKE-UP CALL}

The current COVID-19 pandemic has cast a new light on the translational process from basic academic virology research to prophylactic and therapeutic interventions of safe and efficient antiviral drugs and vaccines in humans. Until recently, virologists were left much unnoticed by the general public in their chambers working on, for the naked eye, invisible infectious agents, keeping viruses such as polio, measles, and mumps at bay with reliable conventional vaccines and producing annual modifications to influenza vaccines to outsmart the novel annual mutant influenza viruses causing outbreaks during the flu season. Then, everything changed! The SARS-CoV-2 outbreak was declared a pandemic by the World Health Organization reaching every corner of the world, and today (February 12, 2021) (https://www.worldometers.info/coronavirus/?\#countries) more than 108 million people have been infected, leading to the death of more than 2.3 million people and the devastation of the global economy. An unprecedented combined effort saw the scientific community, pharmaceutical and biotech companies, and governmental organizations acting together at a level never seen before. The biggest and fastest translational virology program was born!

*Correspondence:

\section{FROM BENCH TO BEDSIDE}

There is no better example of the power of translational medicine, especially translational virology, than what has been illustrated by the record-breaking development of several approved vaccines against SARS-CoV-2. This effort required substantial breakthrough in technology development at all stages from basic research level to clinical applications. In this context, the innovation at the bench, the rapid evaluation in animal models followed by overlapping phase I to III clinical trials and vaccine production at a stage when vaccine safety and efficacy had not been confirmed (1) showed the dedication and the determination of the whole scientific community. It resulted in approval of three different vaccines within more or less a year since the first detection of SARS-CoV-2. Moreover, two additional adenovirus-based vaccines have been approved and more are expected to follow. Although RNA-based vaccines "won the vaccine race," the approval of the simian adenovirus-based vaccine confirmed the power of translational virology. Actually, viral components such as methyl guanosine analogs $(\mathrm{m} 7 \mathrm{G})$ for $5^{\prime}$ end capping of RNA and the formulation of viral-like nanoparticles for the delivery of RNA also played an essential role in RNA vaccine development. Despite the successful rapid development of vaccines against COVID-19, the recent emergence of SARS-CoV-2 variants with enhanced transmissibility and potential pathogenicity (2) has underlined the importance of staying alert by applying translational virology approaches for identification of new mutations in the population and to confirm the efficacy of existing vaccines or engineering new vaccines targeting emerging variants of SARS-CoV-2. 
At the same time as COVID-19 vaccine development was initiated, major efforts to develop antiviral drugs for the treatment of COVID-19 started. Much attention has been paid to repurposing drugs previously developed for other viral infections $(3,4)$. In this context, remdesivir, an inhibitor of RNA-dependent RNA polymerase, which was originally developed for treatment of hepatitis $\mathrm{C}$ virus (HCV), respiratory syncytial virus (RSV), and Ebola virus (EBOV) is evaluated (5). In the case of COVID-19, patients treated with compassionate-use of remdesivir for severe disease showed clinical improvement in $68 \%$ of patients although additional studies are required (6). Obviously, major efforts were dedicated to screening potential novel antiviral drugs (7). Moreover, other antiviral drugs and monoclonal antibodies have been developed including the recently FDA approved casirivimab and imdevimab monoclonal antibodies (http://www. fda.gov/news-events/press-announcements/coronavirus-covid19-update-fda-authorizes-monoclonal-antibodies-treatmentcovid-19).

\section{TRANSLATIONAL VIROLOGY}

Although translational research, and in this case translational virology, has been considered as the process of generating new materials and medical evidence for health care needs, the influence of governmental organizations and authorities plays an important role (8) as has been seen for COVID-19 drug and vaccine development. Translational virology can be divided into areas of basic, preclinical and clinical research, drug production, and drug regulation and approval. Basic research is dominated by activities conducted by academic institutions interested in questions related to basic structure and function of viruses, and rarely present opportunities for directly applied approaches. However, systems biology approaches have provided vast amounts of genomics and proteomics information, which has significantly advanced studies on virushost interactions supporting drug and vaccine development (9). Moreover, complementary short interfering (siRNA) and micro-RNA (miRNA) high-throughput screening technologies and next-generation sequencing have presented a more detailed multidimensional view on virus-host networks. Together with bioinformatics and computational biology, systems biology can have a profound impact on translational research supporting the development of novel therapeutics and vaccines against viral infections. It should also be pointed out that viral vector engineering, which has demonstrated a major impact on both gene therapy and vaccine development, has to a large extent originated from academic institutions (10).

Preclinical studies present a step in the direction toward translational medicine represented by evaluation in mammalian cell lines, primary cell cultures and more recently in threedimensional cell culture models, which range from engineering tissues for clinical applications to development of models for drug screening (11). Tumor models in rodents and primates for the evaluation of cancer drug candidates (12) and verification of vaccine protection in immunized animals after challenges with lethal doses of viral pathogens (13) have been essential parts of translational medicine. To achieve proof-of-concept in mammalian cell lines and animal models has been a prerequisite for proceeding to human clinical trials. However, discrepancies both in innate and adaptive immunity has been described between mouse and human (14). Moreover, mouse models have shown some limitations due to differences in efficacy of gene delivery, their homogenous genetic background and shorter lifespan (15). These findings have resulted in search of models of naturally occurring tumors in larger animals such as dogs (16) also providing the potential for the development of veterinary drugs and vaccines and further to human equivalents as well (17).

Clinical trials represent another step forward to achieve drug and vaccine approval. The immense costs of clinical evaluation have made it possible to carry out studies in humans only with the generous support of pharmaceutical companies or governmental organizations. In this context, the requirements of Good Manufacturing Grade (GMP) material for clinical trials have substantially increased the costs. For this reason, development of safe and efficient viral vectors will contribute to the success making translational virology applications through basic virology innovations an important part of the process. Many examples exist of the engineering of novel safer viral vectors and packaging cell lines, which has facilitated the production of GMP-grade material for clinical trials (18). Moreover, the skyrocketing of the costs of running clinical trials has made it mandatory for thorough preparations starting in the case of translational virology from basic reagents and viral vectors to any procedure applied through the process to the drug or vaccine ready to be administered to patients. Because of the high costs of clinical evaluation, it is essential that a translational vision is applied from the very early first step of drug target selection, viral vector engineering or any other technology.

Drug regulation and approval activities, although hampered by bureaucracy and slow progress, play an important role in drug and vaccine development (8) and present an essential part of translational virology. The demonstration of good safety and efficacy profiles facilitates the task of regulatory bodies to have favorable views on drug approval and fulfillment of bringing therapeutic intervention to the bedside.

\section{EXAMPLE FROM "THE TRANSLATIONAL VIROLOGY INSTRUCTION MANUAL"}

Again, COVID-19 vaccine development represents an excellent example of efficient and accelerated translational virology (19) (Figure 1). Compared to conventional vaccine development, COVID-19 vaccine development was given a head start based on accumulated studies on SARS-CoV (20) and Middle East Respiratory Syndrome-Coronavirus (MERS-CoV) (21). Immunization and toxicology studies gained from the previous experience from SARS-CoV and MERS-CoV and were further accelerated by an aggressive strategy to support a rapid transfer to clinical trials. No time was lost as phase II trials were already initiated based on interim results from phase I studies. Likewise, phase III trials commenced while phase II studies were still in progress. The most astonishing development 


\section{Traditional}

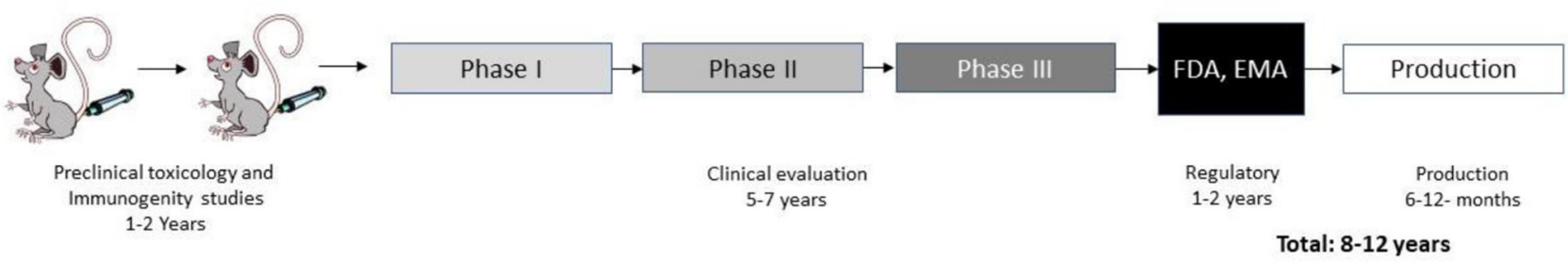

COVID-19

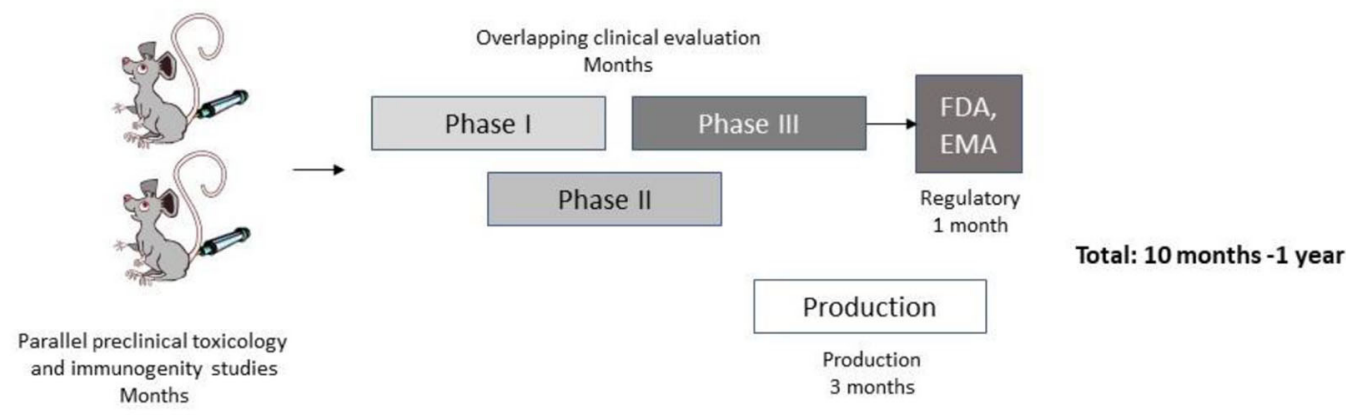

FIGURE 1 | Timelines for traditional and COVID-19 vaccine development.

relates to the production of vaccine candidates while phase III studies were still in progress. This is unprecedented as normally vaccine production only starts when vaccine approval has been received. Obviously, this was a major risk taken, which was made possible by the strong support of the pharmaceutical industry and governmental organizations. This translational virology procedure allowed the long vaccine development process of many years to be shortened to less than a year from initiation to approved vaccine. However, it will most likely be "a once-in-a-lifetime" experience due to the exceptional circumstances. Despite of the unique circumstances, we can learn a lot from the COVID-19 vaccine development process when taking on other translational virology projects.

\section{THE BEGINNING OF THE END OR THE END OF THE BEGINNING}

Despite the strengths and attractiveness of translational virology, it is also hampered by some weaknesses. The enormous information flow at all levels has created a situation, where expertise is restricted to ever narrower areas. A clinician specialized in sophisticated therapeutic interventions is unable

\section{REFERENCES}

1. Lundstrom K. The Current Status of COVID-19 Vaccines. Front Genome Edit. (2020) 2:579297. doi: 10.3389/fgeed.2020.579297

2. Voysey M, Costa Clemens SA, Madhi SA, Weckx LY, Folegatti PM, Aley $\mathrm{PK}$, et al. Safety and efficacy of the ChAdOx1 nCoV-19 vaccine (AZD1222) to grasp the fine details in genetic engineering and molecular biology. Vice versa, a molecular virologist, even with a basic clinical training is at loss at current treatment methods for patients, which could prove essential in design efforts on the molecular level. In between these extreme ends, scientists have to master recombinant virus and protein production at laboratory and large-scale, technologies related to drug and vaccine formulations, immunization, and other in vivo studies in animal models. For this reason, Translational Virology will play a significant role in bringing together the various disciplines by providing a common forum for communication.

Whether the vaccine availability is the beginning of the end or the end of the beginning of the COVID-19 pandemic, to quote Sir Winston Churchill, time will tell. At least with the inauguration of Frontiers in Virology and Translational Virology, I strongly believe this is the beginning of a new era in science for humankind and I welcome you all to contribute to our future success.

\section{AUTHOR CONTRIBUTIONS}

The author confirms being the sole contributor of this work and has approved it for publication. 
4. Riva L, Yuan S, Yin X, Martin-Sancho L, Matsunaga N, Pache L, et al. Discovering of SARS-CoV-2 antiviral drugs through large-scale compound repurposing. Nature. (2020) 586:113-9. doi: 10.1038/s41586-020-2577-1

5. Tchesnokov EP, Feng JY, Porter DP, Götte M. Mechanism of inhibition of Ebola virus RNA-dependent RNA polymerase by remdesivir. Viruses. (2019) 11:326. doi: 10.3390/v11040326

6. Grein J, Ohmagari N, Shin D, Diaz G, Asperges E, Castagna A, et al. Compassionate use of remdesivir for patients with severe Covid-19. N Engl J Med. (2020) 382:2327-36. doi: 10.1056/NEJMoa2007016

7. Sanders JM, Monogue ML, Jodlowski TZ, Cutrell JB. Pharmacologic treatments for coronavirus disease 2019 (COVID-19): a review. JAMA. (2020) 323:1824-36. doi: 10.1001/jama.2020.6019

8. Kimmelman J, Kesselheim AS. Translational research and the US federal elections. Sci Transl Med. (2016) 8:361ed13. doi: 10.1126/scitranslmed.aah5233

9. Peng X, Chan EY, Li Y, Diamond DL, Korth MJ, Katze MG. Virus-host interactions: from systems biology to translational research. Curr Opin Microbiol. (2009) 12:432-8. doi: 10.1016/j.mib.2009.06.003

10. Lundstrom $\mathrm{K}$. RNA viruses as tools in gene therapy and vaccine development. Genes. (2019) 10:189. doi: 10.3390/genes10030189

11. Haycock JW. 3D cell culture: a review of current approaches and techniques. Methods Mol Biol. (2011) 695:1-15. doi: 10.1007/978-1-60761-984-0_1

12. Cheon DJ, Orsulic S. Mouse models of cancer. Annu Rev Pathol. (2011) 6:95-119. doi: 10.1146/annurev.pathol.3.121806.154244

13. Lehrer AT, Wong TS, Lieberman MM, Johns L, Medina L, Feldmann F, et al. Recombinant subunit vaccines protect guinea pigs from lethal Ebola virus challenge. Vaccine. (2019) 37:6942-50. doi: 10.1016/j.vaccine.2019.06.035

14. Mestas J, Hughes CCW. Of mice and not men: differences between mouse and human immunology. J Immunol. (2004) 172:2731-8. doi: 10.4049/jimmunol.172.5.2731

15. Casal M, Haskins M. Large animal models and gene therapy. Eur J Hum Genet. (2005) 14:266-72. doi: 10.1038/sj.ejhg.5201535
16. Tarone L, Barutello G, Iussich S, Giacobino D, Quaglino E, Buracco P, et al. Naturally occurring tumors in pet dogs as pre-clinical models for cancer immunotherapy. Cancer Immunol Immunother. (2019) 68:1839-53. doi: 10.1007/s00262-019-02360-6

17. Denies S, Sanders NN. Recent progress in canine tumor vaccination: potential applications for human tumor vaccines. Expert Rev Vaccines. (2012) 11:137586. doi: 10.1586/erv.12.104

18. Dasgupta A, Tinch S, Szczur K, Ernst R, Shryock N, Kaylor C, et al. Phase I/II manufacture of lentiviral vectors under GMP in an academic setting. Methods Mol Biol. (2020) 2086:27-60. doi: 10.1007/978-1-0716-014 6-4_3

19. Krammer F. SARS-CoV-2 vaccines in development. Nature. (2020) 586:51627. doi: 10.1038/s41586-020-2798-3

20. Sheahan T, Whitmore A, Long K, Ferris M, Rockx B, Funkhouser B, et al. Successful vaccination strategies that protect aged mice from lethal challenge from influenza virus and heterologous severe acute respiratory syndrome coronavirus. J Virol. (2011) 85:217-30. doi: 10.1128/JVI.01805-10

21. Haagmans BL, van den Brand JM, Raj VS, Volz A, Wohlsein P, Smits $\mathrm{SL}$, et al. An orthopoxvirus-based vaccine reduces virus excretion after MERS-CoV infection in dromedary camels. Science. (2016) 351:77-81. doi: $10.1126 /$ science.aad1283

Conflict of Interest: The author declares that the research was conducted in the absence of any commercial or financial relationships that could be construed as a potential conflict of interest.

Copyright (C) 2021 Lundstrom. This is an open-access article distributed under the terms of the Creative Commons Attribution License (CC BY). The use, distribution or reproduction in other forums is permitted, provided the original author(s) and the copyright owner(s) are credited and that the original publication in this journal is cited, in accordance with accepted academic practice. No use, distribution or reproduction is permitted which does not comply with these terms. 